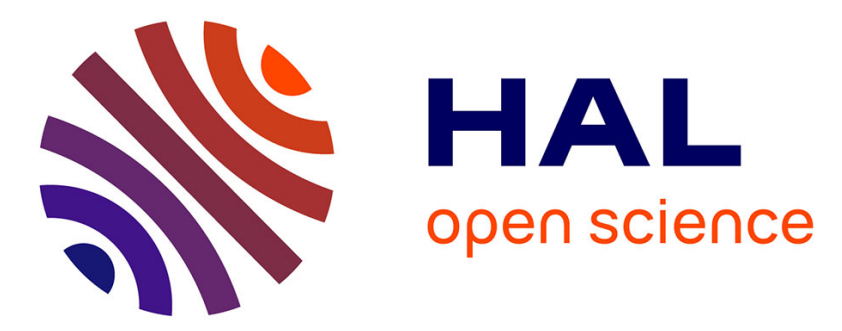

\title{
Statistical tolerance analysis of a hyperstatic mechanism, using system reliability methods
}

Paul Beaucaire, Nicolas Gayton, Emmanuel Duc, Maurice Lemaire, Jean-Yves Dantan

\section{- To cite this version:}

Paul Beaucaire, Nicolas Gayton, Emmanuel Duc, Maurice Lemaire, Jean-Yves Dantan. Statistical tolerance analysis of a hyperstatic mechanism, using system reliability methods. Computers \& Industrial Engineering, 2012, 63 (4), pp.1118-1127. 10.1016/j.cie.2012.06.017 . hal-02517253

\section{HAL Id: hal-02517253 \\ https://hal.science/hal-02517253}

Submitted on 24 Mar 2020

HAL is a multi-disciplinary open access archive for the deposit and dissemination of scientific research documents, whether they are published or not. The documents may come from teaching and research institutions in France or abroad, or from public or private research centers.
L'archive ouverte pluridisciplinaire HAL, est destinée au dépôt et à la diffusion de documents scientifiques de niveau recherche, publiés ou non, émanant des établissements d'enseignement et de recherche français ou étrangers, des laboratoires publics ou privés. 


\title{
Statistical tolerance analysis of a hyperstatic mechanism, using system reliability methods is
}

\author{
Paul Beaucaire ${ }^{\mathrm{a}, *}$, Nicolas Gayton $^{\mathrm{a}}$, Emmanuel Duc $^{\mathrm{a}}$, Maurice Lemaire ${ }^{\mathrm{a}}$, Jean-Yves Dantan ${ }^{\mathrm{b}}$ \\ ${ }^{a}$ Clermont Université, IFMA, EA 3867, Laboratoire de Mécanique et Ingénieries, BP 10448, 63000 Clermont-Ferrand, France \\ ${ }^{\mathrm{b}}$ LCFC, Arts et Métiers, ParisTech Metz, 4 rue Augustin Fresnel, 57078 Metz Cedex 3, France
}

\begin{abstract}
The quality level of a mechanism can be evaluated a posteriori after several months by following the number of warranty returns. However, it is more interesting to evaluate a predicted quality level in the design stage: this is one of the aims of statistical tolerance analysis. A possible method consists of computing the defect probability $\left(P_{D}\right)$ expressed in ppm. It represents the probability that a functional requirement will not be satisfied in mass production. For assembly reasons, many hyperstatic mechanisms require gaps, which their functional requirements depend on. The defect probability assessment of such mechanisms is not straightforward, and requires advanced numerical methods. This problem particularly interests the VALEO W.S. company, which experiences problems with an assembly containing gaps. This paper proposes an innovative methodology to formulate and compute the defect probability of hyperstatic mechanisms with gaps in two steps. First, a complex feasibility problem is converted into a simpler problem. Then the defect probability is efficiently computed thanks to system reliability methods and the $m$-dimensional multivariate normal distribution $\Phi_{m}$. Finally, a sensitivity analysis is provided to improve the original design. The whole approach is illustrated with an industrial case study, but can be adapted to other similar problems.
\end{abstract}

\section{Introduction}

In very competitive industrial fields such as the automotive industry, more and more interest is being paid to the quality level of manufactured mechanisms. It is very important to avoid warranty returns and manage the rate of out-of-tolerance products in production that can lead to assembly line stoppages and/or the discarding of out-of-tolerance mechanisms.

The quality level of a mechanism can be evaluated by the number of faulty parts in production or by the number of warranty returns per year. However, these two methods of product quality evaluation remain a posteriori. Tolerance analysis is a more interesting way to evaluate a predicted quality level in the design stage. Scholtz (1995) proposes a detailed review of classical methods whose goal is to predict functional characteristic variations based on component tolerances. Moreover, statistical tolerance analysis enables the definition of the probability that this functional requirement will be respected, as the well known RSS (Root Sum of Squares) does. Advanced statistical tolerance analysis methods allow the defect probability of an existing design to be computed, knowing the dimension tolerances and functional requirements. Various assumptions about the statistical distributions of component dimensions can be made based on their tolerances. This defect probability, denoted as $P_{D}$ in the following, is expressed in ppm (parts per million) and predicts the number of faulty parts per million in mass production. Several authors have proposed wellestablished methodologies to evaluate this probability for linear (Evans, 1975a) or non-linear analytical expressions (Evans, 1975a; Glancy \& Chase, 1999; Hassani, Aifaoui, Benamara, \& Samper, 2008; Nigam \& Turner, 1995) of functional characteristics.

In many cases, engineers design hyperstatic mechanisms to increase rigidity. For assembly reasons, this kind of mechanism requires functional gaps to remove stresses and fulfill its functions. Often, the functional requirements depend on these gaps. A statistical tolerance analysis of mechanisms containing gaps is not straightforward. In the literature, as Ballu, Plantec, and Mathieu (2008) have noted, hyperstatic mechanisms are rarely studied because of their complexity. Moreover, gaps within mechanisms are often neglected or poorly modeled. Valeo W.S., an automotive company for whom quality management is a top priority, with defect probability goals in ppb (parts per billion), is focused on such a mechanism with functional gaps for which existing methodologies are ineffective or unreliable for several reasons.

This paper proposes an innovative methodology able to compute the defect probability of a hyperstatic mechanism containing 


\section{Nomenclature}

$n \quad$ number of parts

$\mathbf{X} \quad$ vector of part dimensions

$X_{i} \quad i$ th part dimension

$T_{i} \quad X_{i}$ target value

$t_{i} \quad X_{i}$ tolerance

$L S L_{i}, U S L_{i}$ respectively lower and upper specification limits of $X_{i}$

$\sigma_{i} \quad$ standard deviation of $X_{i}$

$\mu_{i} \quad$ mean value of $X_{i}$

$\delta_{i} \quad$ mean shift of $X_{i}$, difference between target and mean values: $\delta_{i}=T_{i}-\mu_{i}$

$\delta_{i}^{(\max )} \quad$ maximum permissible mean shift of $X_{i}$

$C_{p k i}^{(r)}, C_{p i}^{(r)} X_{i}$ capability requirements

$C_{p k i}, C_{p i}$ capability measures of $X_{i}$

$C_{p i}^{(\max )} \quad X_{i}$ maximum capability level obtained in optimal manu-

facturing conditions

g vector of gaps

$g_{1}, g_{2} \quad$ gaps between parts

$f_{c 1}, f_{c 2}$ functional characteristics of the mechanism

$s \quad$ functional requirement threshold (Permissible tightening)

$G\left(X_{i}\right) \quad$ performance function in physical $\mathbf{X}$ space
$U_{i} \quad i$ th part dimension in standard space

$H\left(U_{i}\right) \quad$ performance function in standard $\mathbf{U}$ space

$P_{D} \quad$ defect probability of the mechanism

$\mathrm{C}_{95 \%} \quad 95 \%$ confidence interval of a random result

$P_{j}^{*} \quad$ most probable failure point associated with the $j$ th performance function $G_{j}(\mathbf{X})$

$\beta_{j} \quad$ reliability index associated with the $j$ th performance function $G_{j}(\mathbf{X})$

$\Phi$ cumulative density function of the standard Gaussian distribution

$\chi_{n}^{2} \quad$ cumulative density function of the chi-squared distribution with $n$ degrees of freedom

$\Phi_{m} \quad$ cumulative density function of the $m$-dimensional Gaussian distribution

$m$ number of performance functions

$[\rho] \quad$ covariance matrix

$\boldsymbol{\alpha}^{(j)} \quad$ direction cosines associated with the $j$ th performance function $G_{j}(\mathbf{X})$

$S_{i} \quad X_{i}$ sensitivity index gaps. In the literature focused on this field, either the $P_{D}$ formulation is not adapted to this case study (Ballu et al., 2008; Wu, Dantan, Etienne, Siadat, \& Martin, 2009) or the computation method (Monte Carlo) of the defect probability can be improved (Dantan \& Qureshi, 2009). The proposed methodology includes a particular formulation of $P_{D}$ probability and a computation phase. First, a complex feasibility problem, i.e., the research of the existence of multiple non-negative gaps, is converted into a simpler problem consisting of multiple linear equations. Then $P_{D}$ is efficiently computed thanks to the $m$-dimensional multivariate normal distribution $\Phi_{m}$ originally used in a system reliability method, the FORM (First Order Reliability Method) system. Moreover, this methodology can be applied to other similar problems. In addition, a brief sensitivity analysis is performed in order to improve the quality of the system with a very low increase in manufacturing cost.

In the following section, assembly issues regarding the tolerance analysis of hyperstatic mechanisms containing gaps are illustrated with the particular VALEO W.S. case. A mathematical formulation of the defect probability $P_{D}$ is proposed. Taking into account the complexity of this problem, Section 3 describes three available methods to compute $P_{D}$ including the FORM system one. Two different dimension models, depicting two part manufacturing scenarios, are also proposed. Section 4 compares the different methods and exposes the results of their industrial application. Based on these results, and on a sensitivity analysis, the mechanism is finally redesigned with a very low extra manufacturing cost. Section 5 concludes the paper and presents perspectives for the future.

\section{Hyperstatic mechanisms tolerance analysis for assembly issues}

\subsection{Assembly of a hyperstatic mechanism}

A hyperstatic mechanism is overconstrained. When a part is positioned in space it has six degrees of freedom. It can rotate about the three orthogonal axes and move along each of the three axes. In a mechanism, parts are connected to each other by links which eliminate some of these degrees of freedom. If one or more is eliminated more than once, the parts are overconstrained. This creates stresses, and the mechanism is said to be hyperstatic. This situation appears very often. Most of the time, engineers design such systems to increase rigidity. Sometimes, hyperstatic mechanisms are not desired but endured. These kinds of mechanism often involve assembly problems. For this reason, such mechanisms require functional gaps to remove stresses and fulfill their functions.

These gaps, denoted as $\mathbf{g}$ (vector of gaps) in the following, increase the complexity of statistical analysis. They can neither be directly controlled, nor be considered as random variables. Nevertheless, the gap widths are random variables, although they are not independent and depend upon the independent dimensions variables $X_{i}$ gathered in the vector X. Dantan and Qureshi (2009) introduce the $\exists$ "it exists" quantifier in order to formulate correctly assembly problems concerning mechanisms with gaps. Thus, to ensure mechanism assemblability, at least one feasible gap configuration must be found. The generic defect probability formulation of such a mechanism is:

$\left.P_{D}=\operatorname{Prob} \mathbf{X} \mid \exists \mathbf{g} \in\left[0 ; \mathbf{g}_{\max }(\mathbf{X})\right]: \bigwedge_{i=1}^{m} f_{c i}(\mathbf{X}, \mathbf{g}) \geqslant 0\right)$

where $f_{c i}$ are functional characteristics which generally have to be positive to ensure assemblability, $m$ is the number of functional requirements and $\mathbf{g}_{\max }$ is the vector of gap widths, depending on $\mathbf{X}$ as mentioned previously. In the interests of simplification, subsequent similar equations are written in the following abbreviated form:

$P_{D}=$ Prob $\left.\exists \mathbf{g} \in\left[0 ; \mathbf{g}_{\max }(\mathbf{X})\right]: \bigwedge_{i=1}^{m} f_{c i}(\mathbf{X}, \mathbf{g}) \geqslant 0\right)$

As soon as a gap is involved in a functional characteristic, the problem becomes complex. Two different methods can be used to find a feasible gap configuration. It is possible to consider only extreme gap configurations, as Ballu et al. (2008) and Wu et al. (2009) have done, but this method can neglect certain intermediate situations which play a significant role. To be sure to not 
neglect any of these, it is possible to run an optimization algorithm to determine one feasible gap configuration. This method has been coupled with the Monte Carlo method by Dantan and Qureshi (2009) to compute defect probability. This association of methods has the disadvantage of being very time-consuming.

Section 2.2, describes a VALEO W.S. case study. Section 2.3 shows how the defect probability formulation based on extreme gap configurations can be inadequate when applied to this particular case study. Then, in Section 2.4, an improved formulation is proposed to ensure the assemblability of the VALEO W.S. mechanism. Thanks to this formulation, system reliability methods described in Section 3.2 can be used to compute the defect probability.

\subsection{VALEO W.S. case study}

The problem addressed in this paper is a windshield wiper mechanism designed and manufactured by VALEO W.S.. For confidentiality reasons, only an operating diagram is provided (Fig. 1). It is formed of three different parts, $\mathrm{H}, \mathrm{S}$ and $\mathrm{E}$, whose dimensions are independent random variables and gathered in $\mathbf{X}=\left\{E_{1}, E_{2}, E_{3}, E_{4}\right.$,$E_{5}, H_{1}, H_{2}, H_{3}, S_{1}$ \}. This mechanism is hyperstatic since it requires gaps to assemble it without stresses. Parts $H$ and $E$ are positioned with respect to part $S$. The relative positions of parts $H, E$ and $S$ are determined by the gaps of the mechanism, defined by $\mathbf{g}=\left\{g_{1}, g_{2}\right\}$. These two gaps are not random variables, cannot be directly controlled, and vary between zero and a maximum value; they are shown in Fig. 1:
$0 \leqslant g_{1} \leqslant H_{1}-S_{1}$

$$
0 \leqslant g_{2} \leqslant E_{2}-S_{1}
$$

The operating diagram is deceptive as regards the influence of part orientations. In fact in the real mechanism, which is more compact, orientation defects have no effect. Orientation deviations are therefore ignored in this paper. Two functional characteristics, $f_{c 1}$ and $f_{c 2}$ (Fig. $1 \mathrm{~b}$ ), between parts $H$ and $E$ can be measured. To satisfy the functional requirements of the mechanism, $f_{c 1}$ and $f_{c 2}$ must be greater than a threshold $s$ defining a minimum functional gap if $s$ has a positive value and defining a maximum tightening value if $s$ is negative. If one of these two functional characteristics is lower than $s$, it might aggravate assembly problems, stoppages in the production line and potential functional issues. $f_{c 1}$ and $f_{c 2}$ are functions of $\mathbf{X}$ and $\mathbf{g}$ and are given as follows:

$f_{c 1}(\mathbf{X}, \mathbf{g})=-E_{1}+E_{4}-E_{5}-H_{2}-g_{1}+g_{2}$

$f_{c 2}(\mathbf{X}, \mathbf{g})=-E_{3}+E_{5}+H_{2}+H_{3}+g_{1}-g_{2}$

The aim is to compute the probability (called defect probability, denoted $P_{D}$ ) that the studied mechanism cannot be assembled (i.e. there is contact between parts $H, S$ and $E$ in the detailed zone (Fig. 1b) for $s=0$ ). Practically, the goal is to determine the probability that there exist gap values $\mathbf{g}$ giving rise to both functional characteristics $f_{c 1}$ and $f_{c 2}$ having a value greater than a threshold $s$. The defect probability $P_{D}$ is its complement and reads:

$$
P_{D}=1-\operatorname{Prob}\left[\exists \mathbf{g} \in\left[0 ; \mathbf{g}_{\max }(\mathbf{X})\right]:\left(f_{c 1}(\mathbf{X}, \mathbf{g}) \geqslant s \wedge f_{c 2}(\mathbf{X}, \mathbf{g}) \geqslant s\right)\right]
$$

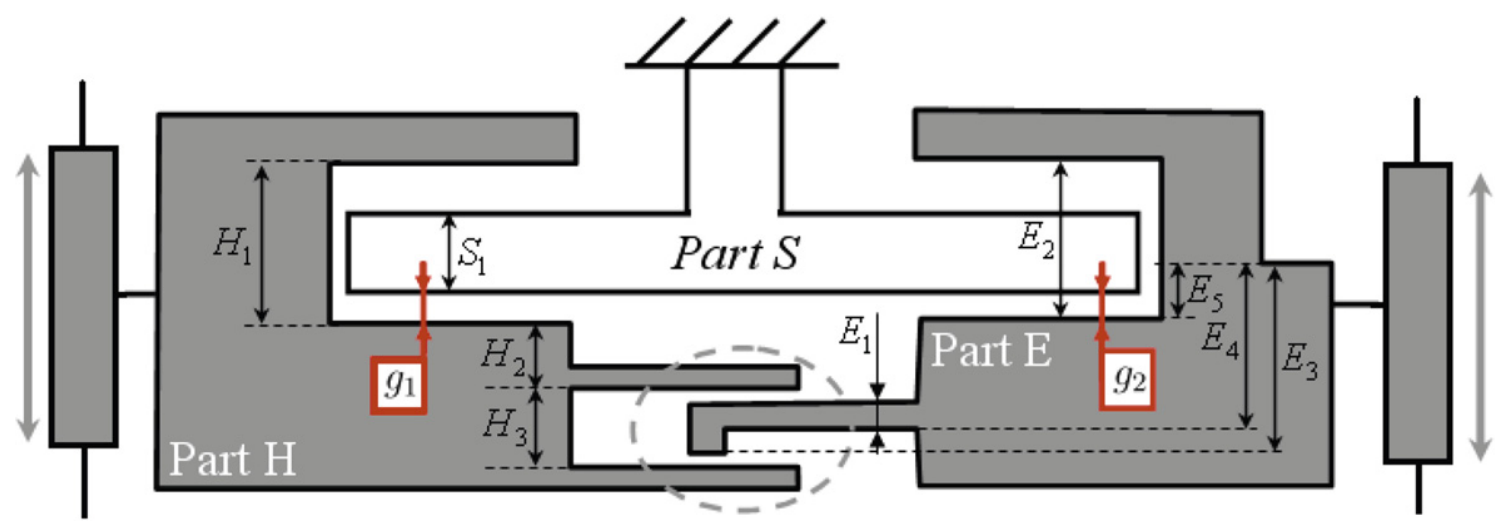

(a) General view

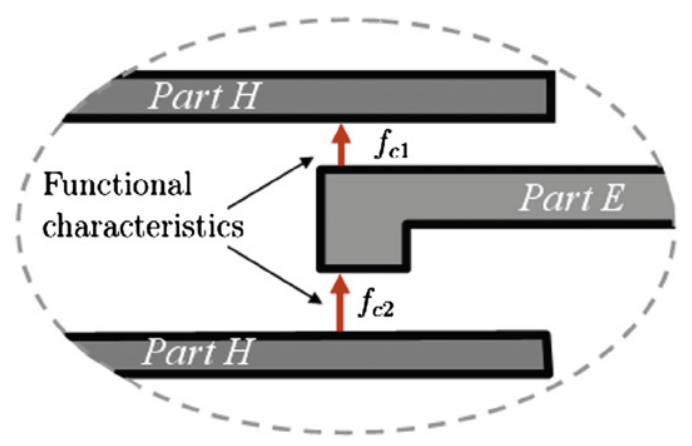

(b) Detailed view

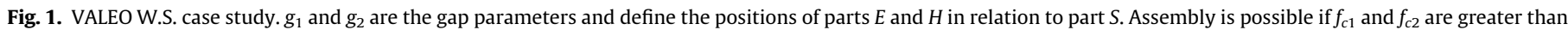
a threshold $s$. 
Table 1

Mechanism parameters $\left\{E_{1}, E_{4}, H_{2}\right\}$ are classical dimensions $\left\{E_{2}, E_{3}, E_{5}, H_{1}, H_{3}, S_{1}\right\}$ are critical ones. $C_{p i}^{(\max )}$ is obtained by monitoring in optimal manufacturing conditions.

\begin{tabular}{llllll}
\hline$X_{i}$ & $T_{i}$ & $t_{i}$ & $C_{p i}^{(r)}$ & $C_{p k i}^{(r)}$ & $C_{p i}^{(\max )}$ \\
\hline$E_{1}$ & 0.7 & 0.2 & 1 & 1 & 2 \\
$E_{2}$ & 1.35 & 0.1 & 1.67 & 1.33 & 2 \\
$E_{3}$ & 3 & 0.2 & 1.67 & 1.33 & 2 \\
$E_{4}$ & 2.4 & 0.1 & 1 & 1 & 2 \\
$E_{5}$ & 0.955 & 0.2 & 1.67 & 1.33 & 2 \\
$H_{1}$ & 1.5 & 0.3 & 1.67 & 1.33 & 2 \\
$H_{2}$ & 0.7 & 0.2 & 1 & 1 & 2 \\
$H_{3}$ & 1.35 & 0.2 & 1.67 & 1.33 & 2 \\
$S_{1}$ & 1.25 & 0.06 & 2 & 2 & 2 \\
\hline
\end{tabular}

It is interesting to note that the expression $f_{c 1}+f_{c 2}=H_{3}-E_{1}-$ $E_{3}+E_{4}$ is independent of $g$ and quantifies the space required by $E$ to be inserted into parts $H$ (see the detailed zone in Fig. 1b). Constraining $f_{c 1}+f_{c 2} \geqslant 2 s$ would be a more convenient way to deal with the problem; unfortunately, it is not sufficient for our purpose. It ensures that there is enough space to assemble the mechanism, but nothing guarantees that the relative positions of parts $H, E$ and $S$, defined by $\mathbf{g}=\left\{g_{1}, g_{2}\right\}$, allow them to fit into place.

Each dimension $X_{i}$ is characterized by a target value $T_{i}$, a tolerance $t_{i}$ and two required capability levels $C_{p i}^{(r)}$ and $C_{p k i}^{(r)}$ (see Table 1 in Section 4 for the values). A manufactured part is in conformance if its measured capability levels $C_{p i}$ and $C_{p k i}$ verify $C_{p i} \geqslant C_{p i}^{(r)}$ and $C_{p k i} \geqslant C_{p k i}^{(r)}$. Capability level expressions are recalled as follows:

$C_{p i}=\frac{t_{i}}{6 \sigma_{i}}$

$C_{p k i}=\frac{\left(t_{i}\right) / 2-\left|\delta_{i}\right|}{3 \sigma_{i}}=\min \left(\frac{\mu_{i}-L S L_{i}}{3 \sigma_{i}} ; \frac{U S L_{i}-\mu_{i}}{3 \sigma_{i}}\right)$

where $\delta_{i}=\mu_{i}-T_{i}$ is the mean shift of $X_{i}, \mu_{i}$ the mean value, $\sigma_{i}$ the standard deviation, $L S L_{i}$ and $U S L_{i}$ respectively the Lower and Upper Specification Limits of $X_{i}$.

\subsection{Defect probability formulation based on the literature}

The complexity of this mechanism is due to mobilities arising from the gaps. To compute the defect probability, the formulation must be independent of uncontrolled variables: the gaps $\mathbf{g}$. This is not the case in Eq. (5). Some authors have dealt with such problems (Ballu et al., 2008; Wu et al., 2009) by considering only extreme gap configurations. This method can be effective for some mechanisms, but not for this particular case study. This subsection proposes a defect probability formulation based on the literature, and illustrates why it is not appropriate.

Considering extreme configurations and following Ballu et al. (2008) and Wu et al. (2009), the mechanism can be assembled if $f_{c 1}$ and $f_{c 2}$ are greater than $s$ in at least one of the four (a, b, c or d) gap configurations in extreme cases (Fig. 2). This assumption allows us to remove the $\mathbf{g}$ variables from Eq. (5) by assigning particular values to the gaps. Let $E^{(i)}$ be the event $\left(f_{c 1}\left(\mathbf{X}, \mathbf{g}^{(i)}\right) \geqslant\right.$ $\left.s \wedge f_{c 2}\left(\mathbf{X}, \mathbf{g}^{(i)}\right) \geqslant s\right),(i)=(\mathrm{a}, \mathrm{b}, \mathrm{c}, \mathrm{d})$. Then $P_{D}$, denoted $P_{D}^{\text {(literature })}$ with this formulation, reads:

$P_{D}^{\text {(literature) }}=1-\operatorname{Prob}\left(E^{(\mathrm{a})} \cup E^{(\mathrm{b})} \cup E^{(\mathrm{c})} \cup E^{(\mathrm{d})}\right)$

As required, this formulation is independent of $\mathbf{g}$.

Thanks to Zou and Morse (2004), it is possible to represent (Fig. 3 ) the gap space of the mechanism in the plane $\left(g_{1}, g_{2}\right)$.

Lines $f_{c 1}=s$ and $f_{c 2}=s$, whose equations are defined in Eqs. (9) and (10), are plotted.

$f_{c 1}=s \Rightarrow-E_{1}+E_{4}-E_{5}-H_{2}-g_{1}+g_{2}=s$

$f_{c 2}=s \Rightarrow-E_{3}+E_{5}+H_{2}+H_{3}+g_{1}-g_{2}=s$

The rectangle represents the numerical limits of $g_{1}$ and $g_{2}$ whose corners are extreme configurations (a, b, c, d) of the mechanism. Thus, the black zone, which is the intersection of the zone between the domains $f_{c 1} \geqslant s$ and $f_{c 2} \geqslant s$ with the rectangle, represents the $\mathbf{g}$ values falling within their bounds and respecting functional requirements. When observing this figure, it is clear that the consideration of only the four extreme cases a, b, c and d is very restrictive, and an infinite number of intermediate configurations would allow a correct assembly. Since there is a black zone, the functional requirements are respected, whereas none of the extreme configurations ( $a, b, c, d$ ) are suitable. This kind of configuration must be taken into consideration. By ignoring intermediate configurations, the formulation based on the literature (Eq. (8)) overvalues the actual defect probability value: $P_{D}^{\text {(literature) }} \geqslant P_{D}$. This defect probability formulation is not appropriate to the present case study.

\subsection{Improved defect probability formulation}

In this subsection, an improved defect probability formulation will be proposed. It is based on graphic considerations.

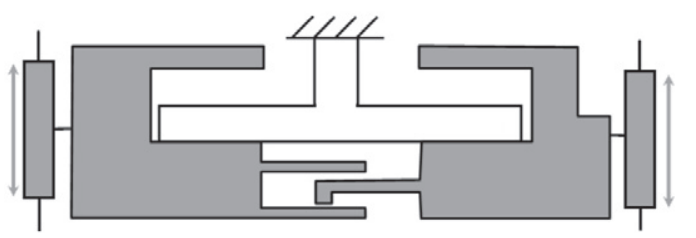

(a) configuration a: $\mathbf{g}^{(\mathrm{a})}=(0,0)$

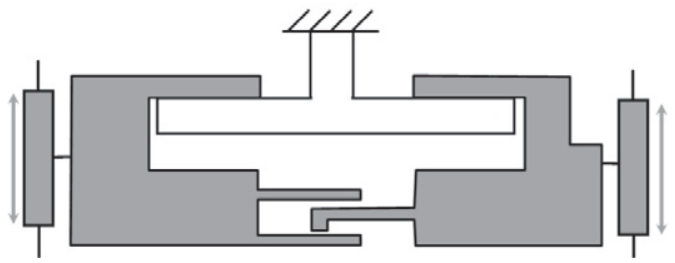

(c) configuration c: $\mathbf{g}^{(\mathrm{c})}=\left(H_{1}-S_{1}, E_{2}-S_{1}\right)$

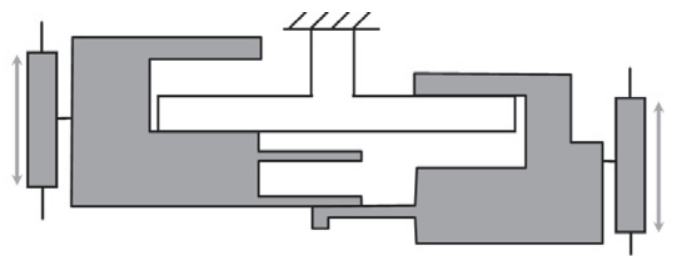

(b) configuration b: $\mathbf{g}^{(\mathrm{b})}=\left(0, E_{2}-S_{1}\right)$

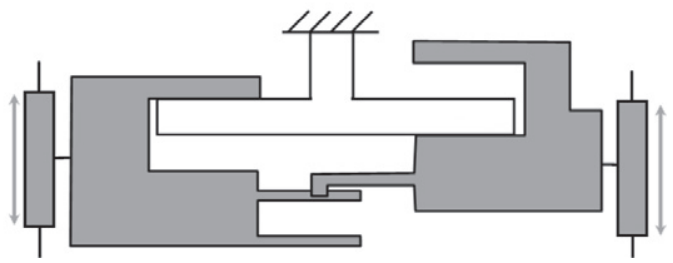

(d) configuration d: $\mathbf{g}^{(\mathrm{d})}=\left(H_{1}-S_{1}, 0\right)$

Fig. 2. Extreme configurations. 


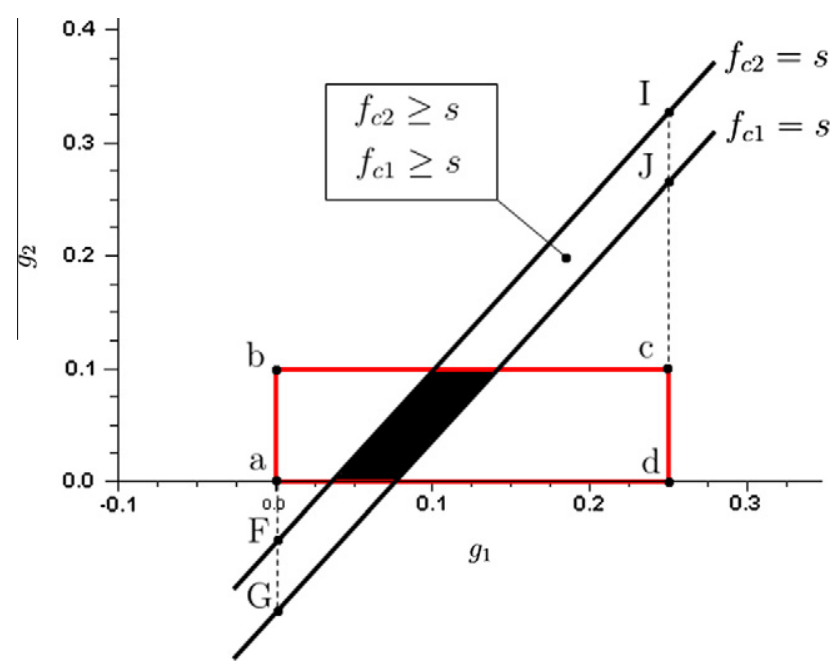

Fig. 3. Gap space representation of the mechanism in a particular configuration. Points a, b, c and d represent extreme gaps configurations. Functional requirements are respected, since the black zone is not empty, whereas no extreme configuration is suitable.

The gap space representation (Fig. 3) enables us to see that only three conditions must be respected to verify the functional requirements:

- Let $\mathrm{G}$ and $\mathrm{J}$ be the points of line $f_{c 1}=s$ corresponding respectively to $g_{1}=0$ and $g_{1}=g_{1}^{\max }$, and $\mathrm{F}$ and $\mathrm{I}$ the points of line $f_{c 2}=s$ corresponding respectively to $g_{2}=0$ and $g_{2}=g_{2}^{\max }$. Since zones $f_{c 1} \geqslant s$ and $f_{c 2} \geqslant s$ are located respectively above and below lines (GJ) and (FI) (see Fig. 3), it is obvious that (FI) must be placed above (GJ) in order to have both functional characteristics greater than $s$ (Eq. (11)).

$$
\begin{array}{ll} 
& -E_{3}+E_{5}+H_{2}+H_{3}-s \geqslant E_{1}-E_{4}+E_{5}+H_{2}+s \\
\text { or } & -E_{1}-E_{3}+E_{4}+H_{3}-2 s \geqslant 0 \\
\text { or } & G_{1}(\mathbf{X}) \geqslant 0
\end{array}
$$

- (FI) should cross the rectangle, so I must be placed above $d$ (Eq. (12)).

$$
\begin{array}{ll} 
& E_{3}-E_{5}-H_{2}-H_{3}+s \leqslant H_{1}-S_{1} \\
\text { or } & -E_{3}+E_{5}+H_{1}+H_{2}+H_{3}-S_{1}-s \geqslant 0 \\
\text { or } & G_{2}(\mathbf{X}) \geqslant 0
\end{array}
$$

- (GJ) should cross the rectangle, so G must be placed below $b$ (Eq. (13)).

$$
\begin{array}{ll} 
& E_{1}-E_{4}+E_{5}+H_{2}+s \leqslant E_{2}-S_{1} \\
\text { or } & -E_{1}+E_{2}+E_{4}-E_{5}-H_{2}-S_{1}-s \geqslant 0 \\
\text { or } & G_{3}(\mathbf{X}) \geqslant 0
\end{array}
$$

It would be possible to do without the gap space representation to define these three conditions by using only Eqs. (1)-(4). Functional characteristics $f_{c 1}\left(g_{1}, g_{2}\right)$ and $f_{c 2}\left(g_{1}, g_{2}\right)$ must be at least greater than $s$ in the most favorable configuration:

$g_{1}=0$ and $g_{2}=E_{2}-S_{1}$ for $f_{c 1}$

$g_{1}=H_{1}-S_{1}$ and $g_{2}=0$ for $f_{c 2}$

This gives the two next conditions, already defined in Eqs. (12) and (13):

$f_{c 1}\left(0, E_{2}-S_{1}\right)=-E_{1}+E_{2}+E_{4}-E_{5}-H_{2}-S_{1} \geqslant s$

$f_{c 2}\left(H_{1}-S_{1}, 0\right)=-E_{3}+E_{5}+H_{1}+H_{2}+H_{3}-S_{1} \geqslant s$

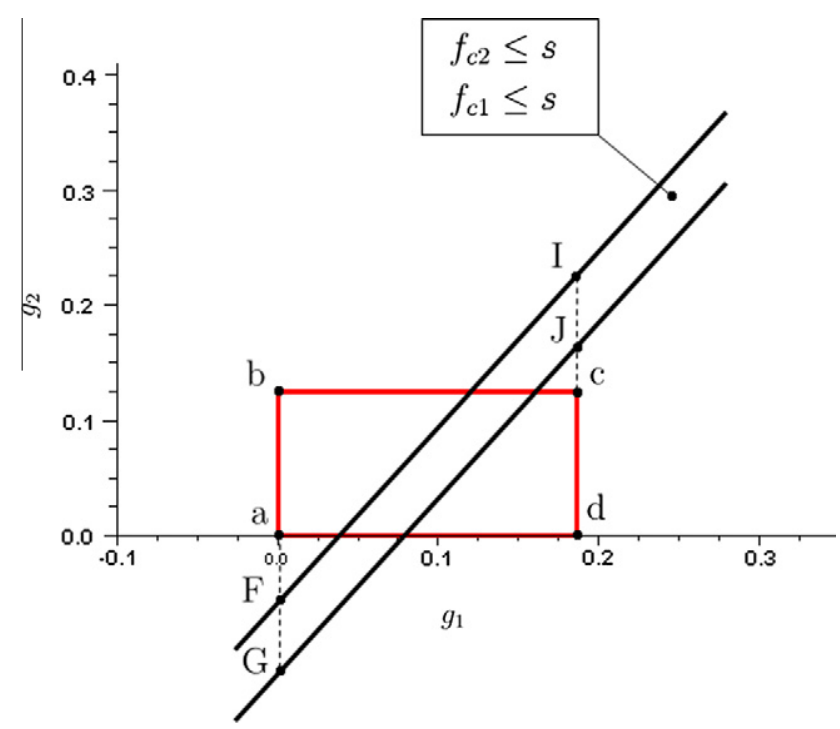

Fig. 4. Gap space representation of the mechanism in a particular configuration Functional requirements are not respected, since there is no black zone.

By combining the two functional requirements $f_{c 1} \geqslant s$ and $f_{c 2} \geqslant s$, we obtain the last condition, already defined in Eq. (11):

$$
\begin{gathered}
f_{c 1}+f_{c 2} \geqslant 2 s \\
\text { or } \quad-E_{1}-E_{3}+E_{4}+H_{3} \geqslant 2 s
\end{gathered}
$$

This is how the three conditions of Eqs. (11)-(13) are obtained analytically. Finally, based on the three performance functions $G_{j}(\mathbf{X}), j=1,2,3$, a new formulation is proposed (Eq. (14)), still independent of $\mathbf{g}$ and taking into account intermediate gap configurations. It is composed only by the intersection of events.

$P_{D}=1-\operatorname{Prob}\left(G_{1}(\mathbf{X}) \geqslant 0 \wedge G_{2}(\mathbf{X}) \geqslant 0 \wedge G_{3}(\mathbf{X}) \geqslant 0\right)$

If one (at least) equation among the three is negative, the mechanism cannot be assembled. In Fig. 4 , the $\mathbf{X}$ dimensions lead to one negative performance function: $G_{1}(\mathbf{X})<0$. So no black zone appears in the gap space representation. This particular mechanism is not functional.

To compute this defect probability (Eq. (14)), two problems arise:

- Each dimension is modeled by random variables, depending on its characteristics. Which statistical properties of $X_{i}$ have to be considered in this probability evaluation? This point will be discussed in Section 3.1 and some proposals will be made.

- How can the intersection probability of two or more events be computed? Two basics probability computation methods are detailed in Section 3.2. Then Section 3.3 present methods able to deal with event intersections. Finally, some proposals are made.

\section{Proposed solutions to deal with defect probability computations}

\subsection{Probabilistic model}

To compute defect probabilities, assumptions about statistical models must be made. In the following, two different models will be considered. First, a non-shifted Gaussian scheme will be considered. Distributions will be centered on their tolerances and $C_{p i}=C_{p i}^{(r)}$ (see Fig. 5a). This hypothesis is optimistic but is the most commonly used (Ballu et al., 2008; Glancy \& Chase, 1999). Second, 


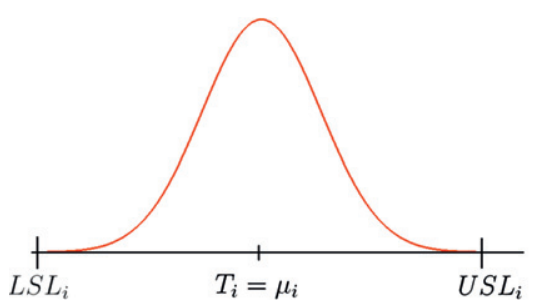

(a) Hypothesis $1 . \mu_{i}=T_{i}, \sigma_{i}=\frac{t_{i}}{6 C_{p i}^{(r)}}$.

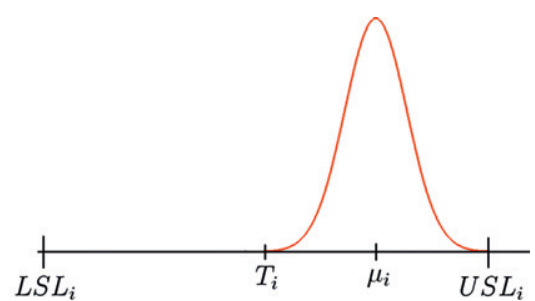

(b) Hypothesis 2 .

$\mu_{i}=T_{i} \pm \frac{t_{i}}{2}\left(1-\frac{C_{p k i}^{(r)}}{C_{p i}^{(m a x)}}\right)$

$\sigma_{i}=\frac{t_{i}}{6 C_{p i}^{(\max )}}$

Fig. 5. Stochastic model.

Gaussian distributions will be shifted by the maximum permissible mean shift $\delta_{i}^{(\max )}$. The sign of each mean shift is found in order to generate the worst combination regarding $P_{D}$, as in Fig. $5 \mathrm{~b}$. The maximum permissible mean shift $\delta_{i}^{(\max )}$ can be reached in the best manufacturing conditions, defined by an upper value of $C_{p i}$ denoted $C_{p i}^{(\max )}$ Gayton et al. (2011), i.e. when the standard deviation of the process is the lowest. So $C_{p i}=C_{p i}^{(\max )}$ and $\sigma_{i}^{(\min )}=\frac{t_{i}}{6 C_{p i}^{(\max )}}$, where $t_{i}$ are dimensions tolerances. Then the capability index $C_{p k i}$ is bounded by $C_{p k i}^{(r)}=\frac{t_{i} / 2-\delta_{i}^{(\max )}}{3 \sigma_{i}^{(\min )}}$. By transforming this expression, the maximum permissible mean shift is obtained: $\delta_{i}^{(\max )}=$ $\frac{t_{i}}{2}\left(1-\frac{C_{p k i}^{(r)}}{C_{p i}^{(m a x)}}\right)$. This second hypothesis, called the statistical worst case, is very pessimistic and accounts for the maximum bound of $P_{D}$. Thus, the impact of the mean shift $\delta_{i}$, which is considered as crucial by Evans (1975b) and Graves (2001), will be investigated.

\subsection{Probability computation for a single event}

To analyze tolerances on a mechanism governed by a single defect event, various authors (Evans, 1975a; Glancy \& Chase, 1999; Nigam \& Turner, 1995) have published methods to compute the following probability:

$P=\operatorname{Prob}(G(\mathbf{X}) \geqslant 0)$

Two methods are described in this section: the well known Monte Carlo method and the FORM (First Order Reliability Method) one, which is available from structural reliability theory (Ditlevsen \& Bjerager, 1986; Lemaire, 2009).

The Monte Carlo simulation method (Evans, 1975a; Lemaire, 2009 ) is used as a reference and can always be used when performance functions can be evaluated economically. The idea is to compute the proportion of successful runs (performance function positive) over the total number of runs $N$. The Monte Carlo simulation gives an estimation of the probability and its associated confidence interval depending on $N$.

The approximation method is called FORM. It requires to transform physical variables $X_{i}$ into standard ones $U_{i}$ thanks to an isoprobabilistic transformation $T$. In the case of uncorrelated Gaussian variables (as these case study variables are), whom standard deviations and mean are respectively denoted $\sigma_{i}$ and $\mu_{i}$, the transformation is direct: $U_{i}=\frac{X_{i}-\mu_{i}}{\sigma_{i}}$. In other cases this can be more complicated (see Ditlevsen \& Bjerager (1986) and Lemaire (2009) for details). In the new $\mathbf{U}$ space, the function $G(\mathbf{X})$ becomes $H(\mathbf{U})=G\left(T^{-1}(\mathbf{U})\right)$. Let $F$ and $K$ be the following two complementary domains:

$F=\{\mathbf{X}: G(\mathbf{X}) \leqslant 0\}=\{\mathbf{U}: H(\mathbf{U}) \leqslant 0\}$
$K=\{\mathbf{X}: G(\mathbf{X}) \geqslant 0\}=\{\mathbf{U} \cdot H(\mathbf{U}) \geqslant 0\}$

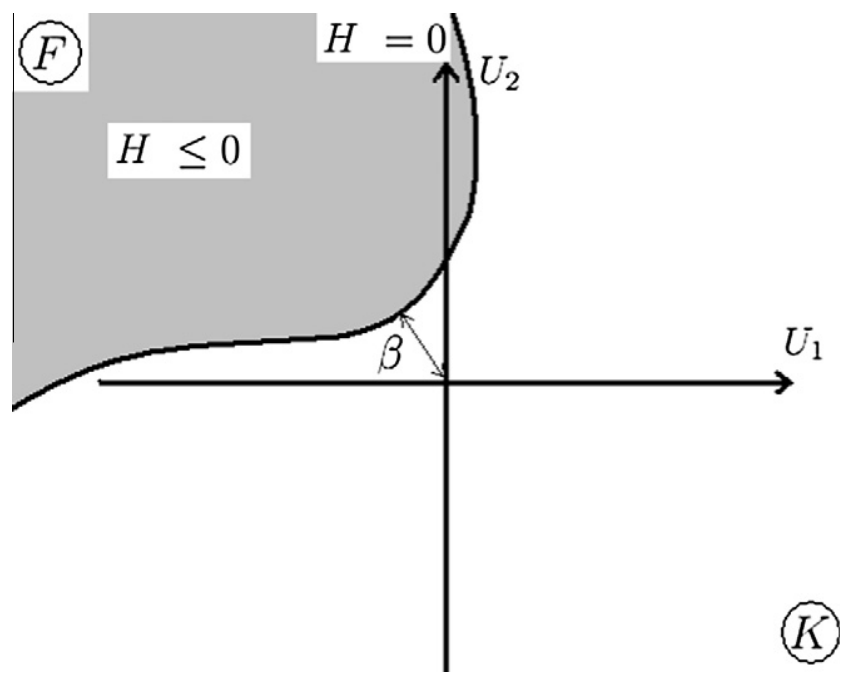

Fig. 6. FORM approach. Representation of actual regions $F$ and $K$. $\mathbf{U}$ is the standard space. $\beta$ is the reliability index.

For illustration purposes, Fig. 6 arbitrarily represents domains $F$ in gray and $K$ in white in a 2-dimensional standard $\mathbf{U}$ space. The reliability index $\beta$ is defined as the minimal distance in the standard $\mathbf{U}$ space between the origin and the domain $H \leqslant 0$. It is obtained by solving the following optimization problem:

$\beta=\min _{\mathbf{U}} \sqrt{\mathbf{U}^{t} \mathbf{U}}$ under the constraint $H(\mathbf{U}) \leqslant 0$

Several algorithms exist to deal with this problem. The most used is the iHLRF (improved Hasofer-Lind-Rackwitz-Fiessler) one (Zhang \& Der Kiureghian, 1995). The point, at the distance $\beta$ of the origin and belonging to $H \leqslant 0$, denoted $P^{*}$, is the most important point in terms of probability and is called "Most Probable failure Point" (MPP) (Fig. 7). At this point, the density probability is the greatest in the $F$ domain. Thus, the original limit-state $H(\mathbf{U})=0$ is replaced by a hyper-plane (a straight line in two dimensions) $\widetilde{H}(\mathbf{U})=0$ at this $\mathrm{P}^{*}$ point. The $K$ region is substituted by the approximated one $\widetilde{K}$ (Fig. 7) and the associated approximated probability $\widetilde{P}$ whose formulation is given in Eq. (16) is considered close to $P$.

$\widetilde{P}=\operatorname{Prob}(\widetilde{G}(\mathbf{X}) \geqslant 0)=\operatorname{Prob}(\widetilde{H}(\mathbf{U}) \geqslant 0)$

$\widetilde{P}$ is evaluated thanks to the cumulative density function of the standard Gaussian distribution:

$P \approx \widetilde{P}=\Phi(\beta)$

If the performance function is a linear combination of Gaussian variables, the limit-state $H(\mathbf{U})=0$ in the standard space is linear. So the evaluated probability is exact $(P=\widetilde{P}=\Phi(\beta))$. In the case of 


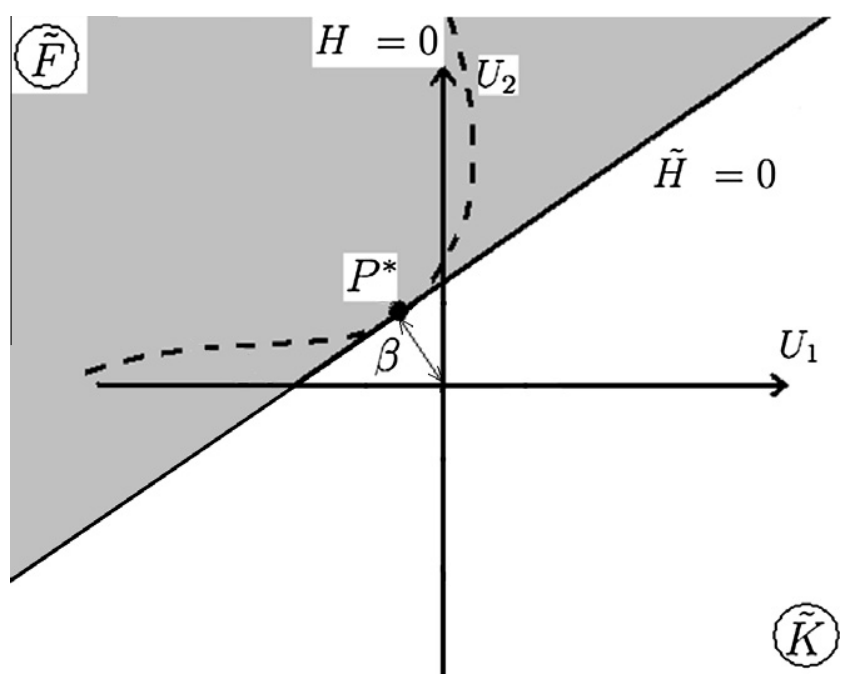

Fig. 7. FORM approach. Representation of approximated regions $\widetilde{F}$ and $\widetilde{K}$. $\mathbf{U}$ is the standard space. $\beta$ is the reliability index.

non-Gaussian variables, the iso-probabilistic transformation is more complicated (see Ditlevsen \& Bjerager (1986) and Lemaire (2009) for details) and $H(\mathbf{U})=0$ is no more linear. Thus, $\widetilde{P}=\Phi(\beta)$ is only an approximation of $P$ defined in Eq. (15).

\subsection{Probability computation for event intersection}

The proposed case study we are dealing with is a "system" problem because its conformity depends on more than one condition. For the proposed formulation, it has been shown that the defect probability of the VALEO W.S. mechanism is governed by the intersection of three events. Moreover, these events are interdependent, since their variables are common. This kind of problem requires advanced numerical methods. Several authors have dealt with system problems: Ballu et al. (2008), Nakanishi and Nakayasu (2002), Savage, Tong, and Carr (2006), and Wu et al. (2009). Let us introduce the general probability formulation:

$P=\operatorname{Prob}\left(\wedge_{j=1}^{m} G_{j}(\mathbf{X}) \geqslant 0\right)=\operatorname{Prob}\left(\wedge_{j=1}^{m} H_{j}(\mathbf{U}) \geqslant 0\right)$

A selection of the existing methods to compute the $P$ probability defined in Eq. (17) are described in this section, and include the Monte Carlo and the FORM methods.

The Monte Carlo simulation method is able to deal with system problem easily. The only difference with the original method is that all performance functions have to be simultaneously positive to get a successful run. The $P$ probability is still the proportion of successful runs over the total number of runs $N$.

The second method is the system version of FORM. It enables the estimation of the linearized problem:

$\widetilde{P}=\operatorname{Prob}\left(\wedge_{j=1}^{m} \widetilde{G}_{j}(\mathbf{X}) \geqslant 0\right)=\operatorname{Prob}\left(\wedge_{j=1}^{m} \widetilde{H}_{j}(\mathbf{U}) \geqslant 0\right)$

Domains $F$ and $K$ in the system case (see Fig. 8) are defined as:

$$
\begin{aligned}
& F=\left\{\mathbf{X}: \bigwedge_{j=1}^{m} G_{j}(\mathbf{X}) \leqslant 0\right\}=\left\{\mathbf{U}: \bigwedge_{j=1}^{m} H_{j}(\mathbf{U}) \leqslant 0\right\} \\
& K=\left\{\mathbf{X}: \bigwedge_{j=1}^{m} G_{j}(\mathbf{X}) \geqslant 0\right\}=\left\{\mathbf{U}: \bigwedge_{j=1}^{m} H_{j}(\mathbf{U}) \geqslant 0\right\}
\end{aligned}
$$

Their representation and their approximations $\widetilde{F}$ and $\widetilde{K}$ are respectively given in Eqs. (8) and (9). Once all reliability indexes $\beta_{j}(j=1$ to $m)$ are determined, the cumulative density function of

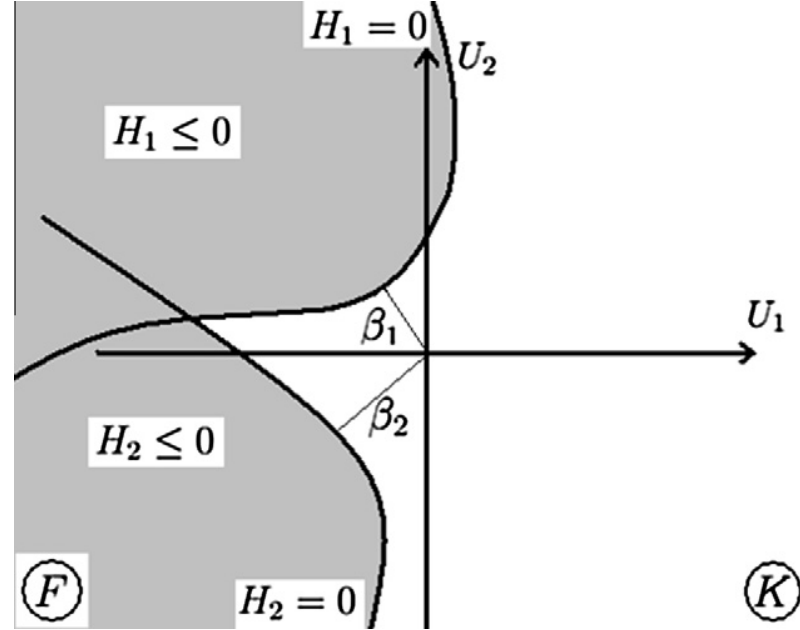

Fig. 8. Representation of actual regions $F$ and $K$ in the system case. $\mathbf{U}$ is the standard space. $\beta_{i}$ are the reliability indexes.

the $m$-dimensional Gaussian distribution $\Phi_{m}$ enables the computation of $\widetilde{P}$ :

$$
\begin{aligned}
\widetilde{P} & =\Phi_{m}\left(\left\{\beta_{1}, \beta_{2}, \ldots, \beta_{m}\right\},[\rho]\right) \\
& =\int_{-\infty}^{\beta_{1}} \cdots \int_{-\infty}^{\beta_{m}} \frac{1}{(2 \pi)^{m / 2} \sqrt{\operatorname{det}[\rho]}} e^{-\frac{1}{2}\{\mathbf{u}\}^{t}[\rho]^{-1}\{\mathbf{u}\}} d u_{1} \cdots d u_{m}
\end{aligned}
$$

where $[\rho]$ is the covariance matrix of performance functions which enables dependent limit-state functions to be taken into account. $\Phi_{m}$ is a $m$-dimensional integral, numerically computed by the Genz method (Genz, 1992; Nadarajah, 2008), whose result is given with a confidence interval depending on the number of runs. This method is more efficient than usual numerical integration techniques to deal with small or very small probabilities.

The third method was proposed by Lee and Woo (1990) and based on Ditlevsen and Bjerager (1986)'s work. Its aim is to bound the probability $\widetilde{P}$ with fewer calculations than the FORM method requires. The reliability indexes have still to be determined. Then, the boundaries of $\widetilde{P}$ defined in Eq. (18), and consequently of $P$ are given by (Lee \& Woo (1990)):

$$
\begin{aligned}
& \qquad \chi_{n}^{2}\left(\min _{j=1}^{m}\left(\beta_{j}\right)^{2}\right) \leqslant \widetilde{P} \leqslant \Phi\left(\min _{j=1}^{m}\left(\beta_{j}\right)\right) \\
& \text { and consequently } \chi_{n}^{2}\left(\min _{j=1}^{m}\left(\beta_{j}\right)^{2}\right) \lesssim P \lesssim \Phi\left(\min _{j=1}^{m}\left(\beta_{j}\right)\right)
\end{aligned}
$$

where $m$ is the number of performance functions and $\chi_{n}^{2}$ is the cumulative density function of the chi-squared distribution with $n$ degrees of freedom ( $n$ being the number of variables). Concerning the upper bound of $\widetilde{P}$, the probability of the intersection is less than or equal to the smallest component probability. Thus:

$\widetilde{P} \leqslant \Phi\left(\min _{j=1}^{m}\left(\beta_{j}\right)\right)$

The hypersphere of radius $\min _{j=1}^{m}\left(\beta_{j}\right)$ always lies inside $\widetilde{K}$. The lower bound of $\widetilde{P}$ is given by the probability covered by this hypersphere and gives:

$\chi_{n}^{2}\left(\min _{j=1}^{m}\left(\beta_{j}\right)^{2}\right) \leqslant \widetilde{P}$

Each method has advantages and disadvantages. Monte Carlo is accurate, but time-consuming. Conversely, the Lee and Woo method is very fast to compute (only one $\beta_{j}$ computation for each limitstate function) but can be very imprecise, depending on the relative positions of the limit-states. The method gives only an upper 


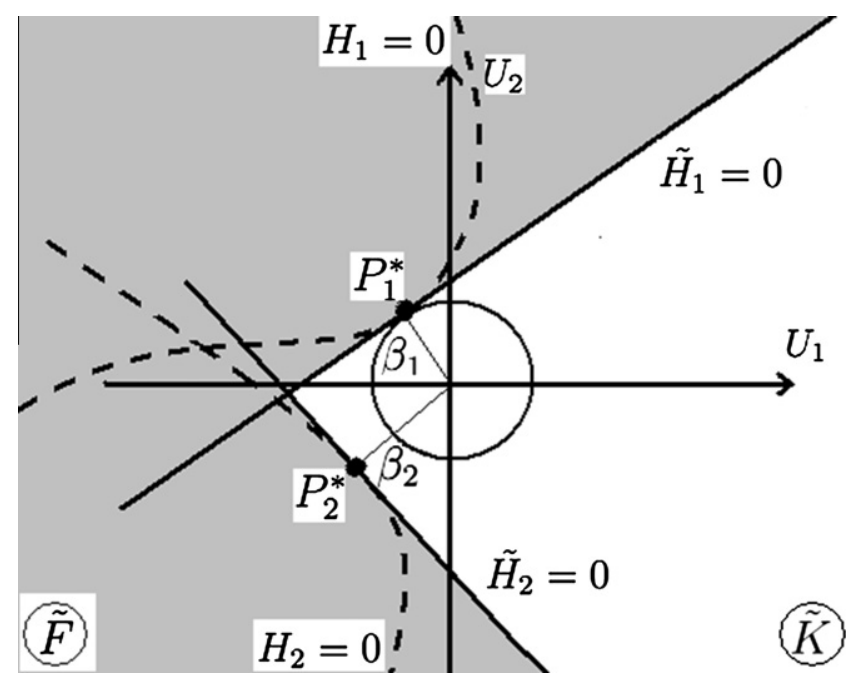

Fig. 9. Representation of approximated regions $\widetilde{F}$ and $\widetilde{K}$ in the system case. $P_{i}^{*}$ are the most probable failure points in relation to the linearized limit-state functions $\widetilde{H}_{j}=0$.

and lower bound of $\widetilde{P}$. It is obvious (see Fig. 9) that the drawn hypersphere badly approximates the $\widetilde{K}$ region. Another uncertainty comes from the first-order approximation between $K$ and $\widetilde{K}$. This approximation is computed at a specific point: the most probable failure point. Nevertheless, experience shows that in most cases, when limit-states are not too curved, the estimation is relevant. The FORM system method using $\Phi_{m}$ is more expensive than the Lee and Woo method, notably when $m$ is large because of the integral computation, but still economical compared to the Monte Carlo method. Finally, we conclude that the FORM system method is a good compromise between computation cost and accuracy. This conclusion will be underlined in the next section, where the three methods will be tested and compared in the industrial case study.

\section{Application to the industrial problem}

\subsection{Tolerance analysis}

The parameters of the industrial case study can be found in Table 1 . Two different kinds of dimensions are presented, classical ones $\left\{E_{1}, E_{4}, H_{2}\right\}$ and critical ones with severe required capability levels $\left\{E_{2}, E_{3}, E_{5}, H_{1}, H_{3}, S_{1}\right\}$. Based on these, the defect probability $P_{D}$ (Eq. (14)) was computed under Hypothesis 1 and 2 described Section 3.1. Details of the calculation for the Lee and Woo and FORM system methods are presented hereafter for the first hypothesis and $s=-0.1$, whereas the Monte Carlo method does not need any details and is only presented to validate the results. In this application, $G_{j}(\mathbf{X})=\sum_{i=1}^{n}\left(a_{i j} X_{i}\right)+b_{j} s$ are linear and the dimensions $\mathbf{X}$ are considered as Gaussian, so $H_{j}(\mathbf{U})$ are linear, $K \equiv \widetilde{K}$ and $P \equiv \widetilde{P}$. Reliability indexes are explicit: $\beta_{j}=\frac{\sum_{i=1}^{n}\left(a_{i j} \mu_{i}+b_{j} s\right)}{\sqrt{\sum_{i=1}^{n}\left(a_{i j} \sigma_{i}\right)^{2}}}$ where $\sigma_{i}=\frac{t_{i}}{6 C_{p i}^{(r)}}$ and $\mu_{i}=T_{i}$ for the first hypothesis. If limit-state functions are non-linear, the FORM method (Lemaire, 2009), based on well-known minimization algorithms, is often able to obtain these reliability indexes in a couple of calls to limit-state functions. Thus, reliability indexes with $s=-0.1$ are:

$$
\left\{\beta_{1}, \beta_{2}, \beta_{3}\right\}=\{5.35,6.25,4.46\}
$$

For the Lee and Woo method, the bounds of $P_{D}$ are given using Eqs. (18) or (19). For the FORM system method, the covariance matrix $[\boldsymbol{\rho}]$ has to be computed beforehand. Let $\boldsymbol{\alpha}^{(i)}=\frac{\nabla \mathbf{H}_{i}\left(\mathbf{U}^{(i) *}\right)}{\left\|\nabla \mathbf{H}_{i}\left(\mathbf{U}^{(i) *}\right)\right\|}$ and $\mathbf{U}^{(i) *}$
Table 2

Hypothesis $1 P_{D}$ bounds in ppm. There is a 95\% chance that $P_{D}$ belongs to the FORM system and Monte carlo method bounds, and a $100 \%$ chance for the Lee and Woo bounds, which are very large.

\begin{tabular}{llll}
\hline & Monte Carlo & FORM system & Lee and Woo \\
\hline$s=-0.1$ & {$[4.20 ; 4.28]$} & {$[3.55 ; 4.86]$} & {$[4.17 ; 18,822]$} \\
$s=-0.05$ & {$[845 ; 847]$} & {$[841 ; 854]$} & {$[667 ; 327,198]$} \\
$s=0$ & {$[143,551 ; 143,565]$} & {$[143,549 ; 143,582]$} & {$[142,425 ; 999,028]$}
\end{tabular}

respectively be the direction cosines relative to the $i$ th limit-state function $H_{i}(\mathbf{U})$ and the $P_{i}^{*}$ point coordinates. According to Lemaire (2009), $\rho_{i, j}=\left\langle\boldsymbol{\alpha}^{(i)} \cdot \boldsymbol{\alpha}^{(j)}\right\rangle$ where $\rho_{i, j}$ and $\langle\bullet \cdot \bullet\rangle$ are the $(i, j)$ th term of the correlation matrix $[\rho]$ and the scalar product. Thus, the $P_{D}$ computation for the first hypothesis, still with $s=-0.1$, is as follows:

$$
\begin{aligned}
P_{D} & =1-\widetilde{P} \\
& =1-\Phi_{3}\left(\{5.35,6.25,4.46\},\left[\begin{array}{ccc}
1 & 0.3 & 0.54 \\
0.3 & 1 & -0.48 \\
0.54 & -0.48 & 1
\end{array}\right]\right)
\end{aligned}
$$

Results for Hypothesis 1, following $s$, are reported in Table 2. For Hypothesis 2, each dimension can be shifted in two different directions as shown in Fig. 5b. To identify the worst case, there is a large number of combinations to study: $2^{n}=2^{9}=512$ in this case. The worst case is the worst combination as regards $P_{D}$ (Eq. (12)). The results are reported in Table 3. The Monte Carlo method provides its $95 \%$ confidence interval width relative to the defect probability, whereas that of the FORM system method is relative to the integration method of $\Phi_{3}$ (Eq. (19)). These two intervals are not strictly comparable but they both indicate that the estimated defect probabilities have a $95 \%$ chance of being contained in these intervals. In the following, they will be considered as equivalent. Thus, the FORM system and Monte Carlo results are expressed in the form of intervals, using their $95 \%$ confidence intervals.

The first remark is that defect probabilities increase when the threshold $s$ increases. This is logical, since the greater the value of $s$, the more severe the functional requirement is. There is every chance that some parts are in contact, while even with $s=0$ for the optimistic hypothesis (Hypothesis 1 ), $P_{D} \approx 144,000 \mathrm{ppm}$. Hypothesis 2 leads to greater defect probabilities than Hypothesis 1, and almost all the parts are faulty with $s=0$. This emphasizes the crucial role that the component mean shifts play in the quality level of the mechanism (Evans, 1975b; Graves, 2001). However, investigation shows that some parts of this mechanism are flexible, so tightening $(s=-0.1)$ is conceivable. With this value, the risk of non-conformance is much lower, but still substantial with the pessimistic Hypothesis 2.

Regarding the methods used to compute $P_{D}$, FORM system results are very close to the Monte Carlo ones, only used as a reference to validate the other methods' results. For this particular problem, limit-state functions are linear, so the FORM system method is exact. Its confidence intervals come from the integration method to compute $\Phi_{m}$. As predicted in the methods presentation section, Lee and Woo bounds are sometimes very far from the actual value, in particular the upper bound, which comes from the hypersphere approximation. Nevertheless, this method has the

Table 3

Hypothesis $2 P_{D}$ bounds in ppm. There are 95\% chances that $P_{D}$ belongs to the FORM system and Monte carlo methods bounds and 100\% chances for the Lee and Woo ones, which are very large.

\begin{tabular}{llll}
\hline & Monte Carlo & FORM system & Lee and Woo \\
\hline$s=-0.1$ & {$[13,724 ; 13,728]$} & {$[13,657 ; 13,779]$} & {$[13,596 ; 844,759]$} \\
$s=-0.05$ & {$[507,483 ; 507,503]$} & {$[507,475 ; 507,818]$} & {$[395,021 ; 1,000,000]$} \\
$s=0$ & {$[999,328 ; 999,329]$} & {$[999,327 ; 999,329]$} & {$[998,900 ; 1,000,000]$} \\
\hline
\end{tabular}




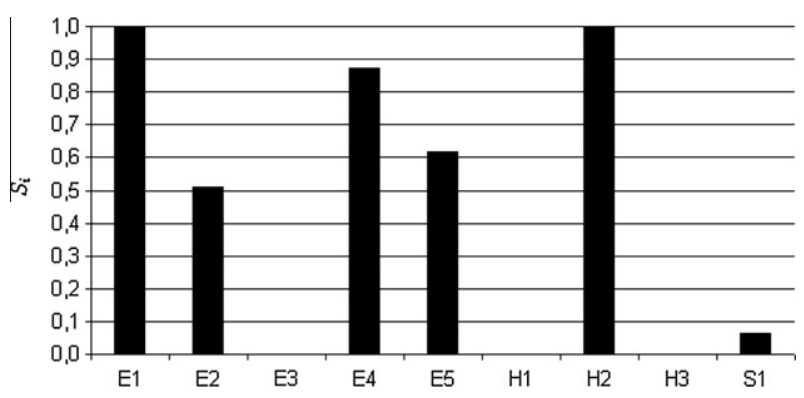

Fig. 10. Sensitivity analysis. $S_{i}$ indexes of $X_{i}$ dimensions for Hypothesis 2 with $s=-0.1$.

advantage of giving results instantaneously. The FORM system method is very accurate and has the advantage of requiring a very low number of calls to limit-state functions; in this industrial case, only one call is needed because the functions are linear. This method, contrary to Monte Carlo, can also deal with problems whose functional characteristics are obtained from a complex CAD code. Indeed, while the Monte Carlo method would need millions of calls to limit-state functions, the FORM system will content itself with only a small number of calls.

\subsection{Sensitivity analysis}

As a consequence, a sensitivity analysis of the defect probability is proposed in order to modify the design to obtain lower defect probabilities, especially with Hypothesis 2 with $s=-0.1$, which is the most pessimistic case. Nominal dimension values and tolerances can be modified to reach target probabilities. Changing nominal values is very effective, but can interfere in other dimensional chains. For this reason, the authors chose to study the influence of tolerances on defect probability. This analysis was performed by finite differences applied using the FORM system method, which enables results to be obtained quasi-instantaneously, under Hypothesis 2 with $s=-0.1$. The results are presented in Fig. 10, where the following normalized sensitivity indexes are computed:

$S_{i}=\frac{1}{\max _{i=1}^{n} S_{i}} \frac{\partial P_{D}^{(H y p .2, s=-0.1)}}{\partial t_{i}}$

To improve the design, it was supposed that all dimensions have the same manufacturing cost, but large tolerances are easier to reduce than tight ones. The following rule was therefore applied: decrease by $20 \%$ the three tolerances of the most sensitive dimensions whose tolerances were originally greater than or equal to $0.2 \mathrm{~mm}$. $E_{1}, E_{5}$ and $H_{2}$ tolerances will thus be reduced since their $S_{i}$ indexes are the highest. $E_{3}, H_{1}$ and $H_{3}$ sensitivities are null. This is probably due to the predominance of a particular performance function in the defect probability, and $G_{3}(\mathbf{X})$ does not contain these dimensions, so they have no influence on defect probability in this

Table 4

Improved parameters. The tolerances of dimensions $E_{1}, E_{5}$ and $H_{2}$ have been decreased by $20 \%$.

\begin{tabular}{llllll}
\hline$X_{i}$ & $T_{i}$ & $t_{i}$ & $C_{p i}^{(r)}$ & $C_{p k i}^{(r)}$ & $C_{p i}^{(\max )}$ \\
\hline$E_{1}$ & 0.7 & $\mathbf{0 . 1 6}$ & 1 & 1 & 2 \\
$E_{2}$ & 1.35 & 0.1 & 1.67 & 1.33 & 2 \\
$E_{3}$ & 3 & 0.2 & 1.67 & 1.33 & 2 \\
$E_{4}$ & 2.4 & 0.1 & 1 & 1 & 2 \\
$E_{5}$ & 0.955 & $\mathbf{0 . 1 6}$ & 1.67 & 1.33 & 2 \\
$H_{1}$ & 1.5 & 0.3 & 1.67 & 1.33 & 2 \\
$H_{2}$ & 0.7 & $\mathbf{0 . 1 6}$ & 1 & 1 & 2 \\
$H_{3}$ & 1.35 & 0.2 & 1.67 & 1.33 & 2 \\
$S_{1}$ & 1.25 & 0.06 & 2 & 2 & 2 \\
\hline
\end{tabular}

Table 5

Comparison of $P_{D}$ results in ppm between the original and the improved design for the two hypotheses with $s=-0.1$.

\begin{tabular}{lll}
\hline & Original design & Improved design \\
\hline Hypothesis $1, s=-0.1$ & 4.24 & 0.07 \\
Hypothesis $2, s=-0.1$ & 13,726 & 127 \\
\hline
\end{tabular}

particular configuration (Hypothesis $2, s=-0.1 \mathrm{~mm}$ ). The new tolerances are noted in bold in Table 4. Based on these new parameters, improved results, obtained by the FORM system method, are indicated and compared with the original ones in Table 5. Finally, the defect probability is almost equal to $100 \mathrm{ppm}$ in the statistical worst case configurations, applying a small tightening $(s=-0.1 \mathrm{~mm})$. This new design is now much safer.

\section{Conclusion}

Mechanisms containing gaps are complex because their nonconformance is governed by combinations or intersections of configurations. This requires the use of several interdependent performance functions which increase the mathematical complexity. This paper describes an innovative method from the structural reliability domain. The defect probability $P_{D}$ was computed in the design phase thanks to the FORM system method. Three main problems arise in this calculation:

- The functional requirements depend on part mobility. A preliminary task is to eliminate uncontrolled mobilities in the probability formulation. The method proposed in the literature, taking into account only extreme configurations, is inefficient in this case. An alternative formulation, considering intermediate configurations, was used here. Thus, a complex feasibility problem, i.e., the research of the existence of multiple non-negative gaps, is converted into a simpler problem consisting of multiple linear equations.

- Among the available methods to compute $P_{D}$, two methods are not convincing. The Lee and Woo approach is not accurate enough in this case, and the Monte Carlo method consumes too much time. The FORM system method was chosen, and proves satisfactory. It seems to be the first application of this method to tolerance analysis considering intermediate gap configurations.

- Dimension modeling is a crucial point. It has been shown that mean shifts play an important role in the tolerance analysis results. Thus, two different assumptions were made, allowing us to consider different parts manufacturing scenarios.

Finally, this entire methodology gives the exact defect probabilities $P_{D}$ when the dimensions are assumed to be normally distributed. This gave rise to a reflection on the choice of tolerances, thanks to a sensitivity analysis. Based on this work, one major area of improvement will be to apply this methodology to non-linear problems such as CAD-based mechanisms. In this case, one evaluation of a limit-state function requires the CAD code to be executed, which is very time-consuming. Thus, the efficiency of the FORM method may be highlighted. This work is already in progress and should lead to a future publication.

\section{Acknowledgments}

The authors would like to thank VALEO W.S., and particularly Gilles Petitet, for their cooperation concerning the presented case study. The Auvergne regional council is also gratefully acknowledged for its financial contribution to this work. 


\section{References}

Ballu, A., Plantec, J.-Y., \& Mathieu, L. (2008). Geometrical reliability of overconstrained mechanisms with gaps. CIRP Annals - Manufacturing Technology, 57(1), 159-162.

Dantan, J.-Y., \& Qureshi, A.-J. (2009). Worst-case and statistical tolerance analysis based on quantified constraint satisfaction problems and monte carlo simulation. Computer Aided Design, 41, 1-12.

Ditlevsen, O. D., \& Bjerager (1986). Methods of structural system reliability. Structural Safety, 4, 195-229.

Evans, D. (1975a). Statistical tolerancing: The state of the art. Part ii. Methods for estimating moments. Journal of Quality Technology, 7(1), 1-12.

Evans, D. (1975b). Statistical tolerancing: The state of the art. Part iii. Shifts and drifts. Journal of Quality Technology, 7(2), 72-76.

Gayton, N., Beaucaire, P., Bourinet, J.-M., Duc, E., Lemaire, M., \& Gauvrit, L. (2011). Apta: Advanced probability - based tolerance analysis of products. Mecanique et Industries, 12, 71-85.

Genz, A. (1992). Numerical computation of multivariate normal probabilities Journal of Computational and Graphical Statistics, 141-149.

Glancy, C. G., \& Chase, K. (1999). A second-order method for assembly tolerance analysis. In Proceedings of the ASME design engineering technical conferences.

Graves, S. (2001). Tolerance analysis tailored to your organization. Journal of Quality Technology, 33(3), 293-303.

Hassani, A., Aifaoui, N., Benamara, A., \& Samper, S. (2008). MTthodologie d'analyse et d'optimisation des tolTrances dans un contexte de conception intTgrTe: Tolanalyses. MTcanique E' Industries, 9, 381-395.
Lee, W.-J., \& Woo, T. C. (1990). Tolerances: Their analysis and synthesis. Journal of Engineering for Industry, 112(2), 113-121.

Lemaire, M. (2009). Structural reliability. ISTE/Wiley.

Nadarajah, S. (2008). On the approximations for multinormal integration. Computers \&' Industrial Engineering 54, 705-708.

Nakanishi, S., \& Nakayasu, H. (2002). Reliability design of structural system with cost effectiveness during life cycle. Computers \& Industrial Engineering, 42, 447-456.

Nigam, S. D., \& Turner, J. U. (1995). Review of statistical approaches to tolerance analysis. Computer Aided Design, 27(1), 6-15.

Savage, G., Tong, D., \& Carr, S. (2006). Optimal mean and tolerance allocation using conformance-based design. Quality and Reliability Engineering International, 22, 445-472.

Scholtz, F. (1995). Tolerance stack analysis methods. Boeing Technical Report.

Wu, F., Dantan, J.-Y., Etienne, A., Siadat, A., \& Martin, P. (2009). Improved algorithm for tolerance allocation based on monte carlo simulation and discrete optimization. Computers \& Industrial Engineering, 56(4), 1402-1413.

Zhang, Y., \& Der Kiureghian, A. (1995). Two improved algorithms for reliability analysis. In R. Rackwitz, G. Augusti, \& A. Bori (Eds.), Reliability and optimization of structural systems, Proceedings of the 6th IFIP WG7.5 Working Conference on reliability and optimization of structural systems. Chapman \& Hall.

Zou, Z., \& Morse, E. (2004). A gap-based approach to capture fitting conditions for mechanical assembly. Computer Aided Design, 36, 691-700. 